\title{
Extracorporeal Membrane Oxygenation for the Support of a Potential Organ Donor with a Fatal Brain Injury before Brain Death Determination
}

\author{
Sung Wook Chang, M.D. ${ }^{1,2}$, Sun Han, M.D. ${ }^{2}$ Jung Ho Ko, M.D., Ph.D. ${ }^{3}$, and Jae-Wook Ryu, M.D., Ph.D. ${ }^{2}$ \\ ${ }^{1}$ Trauma Center, ${ }^{2}$ Department of Thoracic and Cardiovascular Surgery, ${ }^{3}$ Department of Neurosurgery, Dankook University Hospital, Cheonan, Korea
}

The shortage of available organ donors is a significant problem and various efforts have been made to avoid the loss of organ donors. Among these, extracorporeal membrane oxygenation (ECMO) has been introduced to help support and manage potential donors. Many traumatic brain injury patients have healthy organs that might be eligible for donation for transplantation. However, the condition of a donor with a fatal brain injury may rapidly deteriorate prior to brain death determination; this frequently results in the loss of eligible donors. Here, we report the use of venoarterial ECMO to support a potential donor with a fatal brain injury before brain death determination, and thereby preserve donor organs. The patient successfully donated his liver and kidneys after brain death determination.

Key Words: extracorporeal membrane oxygenation; brain death; organ donation.

Organ transplantation has become an effective modality for the treatment of end-stage organ failure, such as that observed in renal and hepatic diseases. Living donors are preferred, following the identification of suitable donors, as delayed graft function has a lower incidence rate in living-donor transplantation.[1-3]

Unfortunately, the shortage of available donor organs is a significant problem that has manifested as a result of a deficit between demand and supply and the relatively small fraction of patients that meet the strict criteria for brain death.[2,4] We report a case of successful extracorporeal membrane oxygenation (ECMO) in a potential donor before brain death determination; the donor had sustained a fatal brain injury. The organs were successfully preserved and donated after brain death determination.

\section{Case Report}

A 67-year-old man with unremarkable medical history was admitted to the emergency department of a local hospital after a motorcycle accident. Non-enhanced computed tomography (CT) of the brain revealed subdural hemorrhage in the bilateral

Received on March 21, 2016 Revised on May 12, 2016

Accepted on May 13, 2016

Correspondence to: Jae-Wook Ryu, Department of Thoracic and Cardiovascular Surgery, Dankook University Hospital, 201 Manghyang-ro, Dongnam-gu,

Cheonan 31116, Korea

Tel: +82-41-550-6195, Fax: +82-41-550-7050

E-mail: j3thorax@chol.com

*No potential conflict of interest relevant to this article was reported. tentorium and the left frontoparietotemporal and right frontal areas, with midline shifting to right. Interventricular hemorrhage in the fourth and both lateral ventricles was also observed.

He was intubated and subsequently referred to our trauma center where he went into cardiac arrest upon arrival. Cardiac massage was performed and spontaneous circulation was 
restored after $3 \mathrm{~min}$. Following this, he was in a comatose state, with a Glasgow Coma Scale of $2 \mathrm{~T}$ and fixed pupils at $3 \mathrm{~mm}$. A follow-up brain CT revealed bilateral subarachnoid hemorrhage with the progression of subdural hemorrhage and brain edema. We decided to perform an emergency craniectomy, which revealed massive hematoma and active bleeding foci in the subdural space. Severe swelling of the brain was observed following removal of the subdural hematoma. Bleeding was controlled with bipolar electrocautery and compression using a hemostatic agent. The skull flap could not be replaced due to the severe brain edema. After duroplasty with artificial dura and scalp closure, the patient was transferred to the intensive care unit (ICU). Although the patient remained hemodynamically stable, the brain stem reflex was, unfortunately, undetectable.

The patient was a potential organ donor who met the strict criteria for brain death. His family agreed to withdraw ICU support, and was informed of the possibility of organ donation after brain death. The specialists who evaluated potential donor and declared brain death were contacted 14 $\mathrm{h}$ postoperatively. Unfortunately, the patient's hemodynamic status began to rapidly deteriorate, despite the maximal doses of inotropic agents; the mean arterial pressure and oxygen saturation were under $40 \mathrm{mmHg}$ and $80 \%$, respectively. At that moment, his vital signs indicated that survival until brain death determination was unlikely. After deliberation with the patient's family, we decided to perform ECMO (Maquet Rotaflow Centrifugal Pumps with Quadrox-D oxygenators, Maquet, Rastatt, Germany) for organ preservation before brain death determination; we also attempted to identify a suitable recipient.

ECMO was promptly established using the Seldinger technique at the ICU bedside. ECMO support was initiated using the venoarterial method via the femoral route, with biocoated circuits. The circuit had double access through a 17-Fr arterial catheter and a 19-Fr venous catheter (Biomedicus $^{\mathrm{TM}}$, Medtronic, Minneapolis, MN, USA). Sodium bicarbonate and heparin were infused to maintain $\mathrm{pH}>7.2$ and an activated clotting time $>180 \mathrm{~s}$; adjustments of arterial blood gases and serum electrolytes were appropriately made based on these values, using normothermic perfusion.

After ECMO, mean arterial pressure and saturation increased to $70 \mathrm{mmHg}$ and $>90 \%$, respectively. The ECMO support oxygenator and sweep gas flow were turned off dur- ing routine brain death determination, which was conducted twice. During the apnea test, it was observed that the patient's hemodynamic status was not deteriorated. Ten hours later, the patient was declared brain dead and was transported to the operating room for the procurement of abdominal solid organs. Procurement of the liver and two kidneys was performed. Subsequently, these organs were successfully transplanted to three individual recipients.

\section{Discussion}

Although organ transplantation is an effective treatment for end-stage organ failure, it is limited by the availability of viable organs.[1,5] According to the Korean Network for Organ Sharing (KONOS) annual report in 2014, patients on the transplantation waiting list required a total of 24,607 organs, including 14,477 kidneys and 4,422 livers. However, only 2,471 donors provided 3,901 organs (kidneys [n $=1,808]$ and livers $[\mathrm{n}=1,262])$.[6] Therefore, many doctors aggressively manage organ donors in order to increase the number of organs available for transplantation. Among the various management techniques currently used, ECMO support for potential organ donors with hemodynamic instability holds potential as a treatment modality and has been shown to provide acceptable results.[5,7-8] In South Korea, several reports associated with ECMO for management of potential donor were published.[9-11]

Trauma patients are often young and healthy; of these, severe trauma patients who sustain fatal and irreversible neurological injuries may be eligible for organ donation.[2] If the patients' families legally consent to the withdrawal of life support and if the patient has provided prior consent for organ donation, physicians make appropriate arrangements for organ donation. However, severe trauma patients are more susceptible to the sudden onset of cardiac arrest. [5] As per a previous report, multiple donations were unsuccessful because the potential donors died immediately after brain death determination. The report went on to recommend early ECMO support to avoid donor loss due to hemodynamic instability and deterioration.[5] This recommendation was implemented much before brain death determination in the present case because the patient's vital signs rapidly deteriorated. Early initiation of ECMO support 
could have led to an extended period of warm ischemia, and subsequent successful organ donation. Physicians may encounter unexpected problems during ECMO. ECMO support may interrupt brain and cardiac death determination due to the partial recovery of brain stem damage and cardiac reanimation, respectively.[5] Moreover, physicians may sometimes find that the patient's hemodynamic status is deteriorated during the apnea test.[12] Therefore, ECMO support should cater to individual patient conditions and be provided in lieu of these in order to avoid unnecessary life support and/or loss of donor organs. For example, thoracic aortic occlusion with balloon catheter insertion and inflation was performed after cardiac death determination in order to prevent cardiac reanimation.[2] Case-specific conditions, like those observed in the present case, should therefore be taken into consideration.

A patient with an unrecoverable brain injury may be a potential organ donor. Brain death and irreversible brain injury are very complex processes that are associated with hemodynamic and metabolic changes. If left untreated, these frequently result in cardiovascular collapse. However, ICU and trauma teams rarely manage these patients aggressively, which can result in the loss of organs available for donation, thereby further exacerbating the shortage of donors. From an ethical and legal standpoint, using ECMO to support a trauma patient with an irreversible brain injury may not be appropriate for patient survival. However, ECMO support is appropriate when attempting to manage an unstable potential donor who is brain dead, as it restores the flow of warm oxygenated blood and may decrease the incidence of delayed graft function. This can result in an expansion of the donor pool. In addition, the cost required for ECMO support associated with organ donation should be paid by national organizations or organ donation should be fully funded.

In conclusion, a patient with an irreversible neurological injury may be eligible for organ donation. Therefore, trauma teams and intensivists should be made aware of the potential of ECMO to aggressively manage potential organ donors before brain death determination. However, there do remain challenges to utilizing ECMO for donor support and management, and the technique requires further development. ECMO should be incorporated as an integral component of the donation process in order to ensure that an increased number of available organs are preserved for transplantation.
ORCID

Sung Wook Chang http://orcid.org/0000-0002-2689-3068

\section{References}

1) Matas AJ, Payne WD, Sutherland DE, Humar A, Gruessner RW, Kandaswamy R, et al: 2,500 living donor kidney transplants: a single-center experience. Ann Surg 2001; 234: 149-64.

2) Magliocca JF, Magee JC, Rowe SA, Gravel MT, Chenault RH, Merion RM, et al: Extracorporeal support for organ donation after cardiac death effectively expands the donor pool. J Trauma 2005; 58: 1095-101; discussion 1101-2.

3) Salim A, Velmahos GC, Brown C, Belzberg H, Demetriades D: Aggressive organ donor management significantly increases the number of organs available for transplantation. J Trauma 2005; 58: 991-4.

4) Ke HY, Lin CY, Tsai YT, Lee CY, Hong GC, Lee CH, et al: Increase the donor pool: transportation of a patient with fatal head injury supported with extracorporeal membrane oxygenation. J Trauma 2010; 68: E87-8.

5) Hsieh CE, Lin HC, Tsui YC, Lin PY, Lin KH, Chang YY, et al: Extracorporeal membrane oxygenation support in potential organ donors for brain death determination. Transplant Proc 2011; 43: 2495-8.

6) Korean Network for Organ Sharing (KONOS): Annual data report. [accessed on October 14, 2015]. Available at https://www.konos.go.kr/konosis/common/bizlogic. jsp.

7) Englesbe MJ, Woodrum D, Debroy M, Chenault R, Miller W, Miskulin J, et al: Salvage of an unstable brain dead donor with prompt extracorporeal support. Transplantation 2005; 79: 378.

8) Barrou B, Billault C, Nicolas-Robin A: The use of extracorporeal membranous oxygenation in donors after cardiac death. Curr Opin Organ Transplant 2013; 18: 148-53.

9) Lee JH, Hong SY, Oh CK, Hong YS, Yim H: Kidney transplantation from a donor following cardiac death supported with extracorporeal membrane oxygenation. J Korean Med Sci 2012; 27: 115-9. 
10) Lee H, Cho YH, Sung K, Yang JH, Chung CR, Jeon K, et al: The use of extracorporeal circulation in suspected brain dead organ donors with cardiopulmonary collapse. J Korean Med Sci 2015; 30: 1911-4.

11) Yang JH, Cho YH, Chung CR, Jeon K, Park CM, Suh GY: Use of extracorporeal membrane oxygenation for optimal organ donation. Korean J Crit Care Med 2014;
29: 194-6.

12) Saucha W, Sołek-Pastuszka J, Bohatyrewicz R, Knapik P: Apnea test in the determination of brain death in patients treated with extracorporeal membrane oxygenation (ECMO). Anaesthesiol Intensive Ther 2015; 47: 368-71. 